\title{
Incidence and predisposing factors of chalazion
}

\author{
Alaa Alsammahi ${ }^{1 *}$, Ziyad Aljohani², Nafisah Jaad ${ }^{3}$, Omamah Abu Daia $^{4}$, Majed Aldayhum ${ }^{4}$, \\ Mohammed Almutairi ${ }^{5}$, Mohammad Basendwah ${ }^{6}$, Reham Alzahrani ${ }^{7}$, Muna Alturki ${ }^{8}$
}

College of Medicine, ${ }^{1}$ King Abdulaziz University, Jeddah, ${ }^{2}$ Ibn Sina National College, Jeddah, ${ }^{3}$ Umm Al-Qura University, Mecca, ${ }^{4}$ King Khalid University, Abha, ${ }^{5}$ Qassim University, Qassim, ${ }^{7}$ Taif University, Taif, Saudi Arabia ${ }^{6}$ Department of Ophthalmology, South Qunfudah Hospital, Qunfudah, Saudi Arabia

${ }^{8}$ Department of Histopathology, King Abdulaziz Hospital, Jeddah, Saudi Arabia

Received: 26 September 2018

Accepted: 13 October 2018

\section{*Correspondence:}

Dr. Alaa Alsammahi,

E-mail: samahi779@gmail.com

Copyright: $\odot$ the author(s), publisher and licensee Medip Academy. This is an open-access article distributed under the terms of the Creative Commons Attribution Non-Commercial License, which permits unrestricted non-commercial use, distribution, and reproduction in any medium, provided the original work is properly cited.

\begin{abstract}
Chalazion is one of the most common eyelid masses seen by ophthalmologists in outpatient clinics. It presents with slowly growing painless cystic lesion affecting eyelids. Chalazion arises due to obstruction of the meibomian gland ducts leading to retained secretion. The exact incidence of chalazion is variable among the studies and it differs depending on certain predisposing factors. Many factors ae known to predispose to chalazion formation including hormonal changes during puberty and pregnancy, viral and bacterial infection, rosacea, exposure to air pollutants, poor eyelid hygiene, chronic blepharitis, seborrheic dermatitis, eyelid trauma, immune deficiency states, eyelid trauma, hyperlipidemia, tuberculosis, and leishmaniasis. This article will review these factors as well as the incidence of chalazion.
\end{abstract}

Keywords: Chalazion, Incidence, Risk factors, Predisposing factors

\section{INTRODUCTION}

Chalazion is one of the most common swellings affecting the eyelid. It is a benign slowly growing cyst that affects upper or lower eyelids, unilaterally or sometimes bilaterally. The word "Chalazion" is a Greek term that means a small pimple. ${ }^{1}$ Chalazion is also known as "Meibomian cyst" because it arises due to chronic sterile granulomatous inflammation of the meibomian gland and its duct leading to retained sebaceous secretions. ${ }^{2}$ Normally, meibomian glands (or tarsal glands) secrete oily sebaceous secretions that spread over the surface of the cornea and conjunctiva to keep them moisture and prevent their dryness. Chalazia arise when the ducts of the tarsal (or meibomian) glands get obstructed. In these cases, the sebaceous secretions accumulate inside the gland forming a progressively growing cyst on the eyelids. ${ }^{3}$
Chalazia present as slowly growing painless cystic lesions on the eyelid. Many of them are tiny, some are visible, and in rare cases they may be large enough to exert pressure manifestations on the eye globe and temporary visual affection (particularly hypermetropia or astigmatism). ${ }^{4-6}$ Patients with chalazion present with eyelid heaviness, conjunctival irritation, sense of dryness, and lacrimation. Sensitivity to light and headache may also occur. ${ }^{7}$ If the chalazion becomes secondarily infected, it will be tender, painful, with discolored overlying skin, and fever may occur. Most of the chalazia are self-limited. However, some cases may need warm compresses, corticosteroid, or surgical evacuation and removal. $^{2,5}$

To date, the exact cause of chalazion formation. Some predisposing factors are known to cause chalazion 
formation. This article will review these factors as well as the incidence of chalazion.

\section{INCIDENCE OF CHALAZION}

The incidence of chalazion is variable among the literature studies. ${ }^{8-11}$ It generally ranges from $0.2 \%$ to $0.7 \%$. In the United States, the exact prevalence is not well-known, but it is commonly encountered among school children and adults between the ages of 30 and 50 years. ${ }^{12}$ In Brazil, the incidence was reported to range from $0.2-0.3 \% .^{12}$ One of the studies conducted in India reported an incidence of $0.24 \% .^{13}$ The incidence in Nigeria was reported to be $0.7 \%$ in one study. ${ }^{14}$

Some studies report a sex difference in the incidence of chalazion with females experiencing higher rates of lesions. $^{1,8}$ This is often attributed to the hormonal influence of sebum production especially during puberty and pregnancy. ${ }^{3}$ However, other researchers did not find a significant difference between both sexes. ${ }^{11,13,15}$ Age was also reported to affect the incidence and prevalence of chalazion. Chalazion occur in all ages, but it occurs maximally in children and adults below the age of 30 years probably due to the higher level of androgenic hormones that stimulate sebum production and viscosity. ${ }^{3,14}$ The mean age of chalazion was reported to be around 25 years, and more than two thirds of the cases were found to occur during the second and third decades of life. ${ }^{16}$ Chalazia are more common in the upper lids due to the higher number of tarsal glands in comparison to the lower lids. ${ }^{17}$

\section{PREDISPOSING FACTORS TO CHALAZION}

Though the pathophysiology of chalazion is well-known, the predisposing factors for meibomian gland dysfunction are less established. Chalazion - as aforementioned occurs due to obstruction of the meibomian gland duct. The exact underlying cause for this obstruction remains elusive. Many factors have been proposed, for example, hormonal changes during puberty and pregnancy, viral and bacterial infection, rosacea, exposure to air pollutants, poor eyelid hygiene, chronic blepharitis, seborrheic dermatitis, eyelid trauma, and diabetes. $3,13-15,18$ Other less associated factors include immune deficiency states, hyperlipidemia, tuberculosis, and leishmaniasis. ${ }^{19,14,20,21}$ These predisposing factors will be reviewed in this section.

\section{Hormonal changes}

Many hormones play a role in secretion of sebum from the meibomian glands. Androgens and androgen receptors are well-known to increase both the secretion and viscosity of the sebaceous glands. ${ }^{22}$ Androgens enhance the growth of all sebaceous glands in the body and regulate their homeostasis. Lipogenesis also increases in the presence of androgens leading to increased viscosity of the produced sebum. ${ }^{23}$ Therefore, chalazia are commonly encountered among adolescents and pregnant women. ${ }^{1}$

\section{Viral and bacterial infection}

Infection of the Meibomian glands and Meibomian gland ducts is another proposed factor for chalazion development. Chalazion was reported to spread among school children, and to be transmitted between family members and household contacts in one report. It was also reported to follow follicular conjunctivitis and systemic infections. ${ }^{15}$ Viral etiology was confirmed by histopathological examination of chalazion Meibomian gland tissue examination. ${ }^{15}$ Bacteria contribute in the pathogenesis of chalazion as well. Certain organisms, particularly staphylococcus aureus, can cause Chalazia, whilst other organisms occur as secondary superimposed infection on top of the already developed chalazion. ${ }^{24}$

\section{Rosacea}

Chalazia were reported to be significantly associated with rosacea. Rosacea is a common inflammatory condition affecting the facial skin. Patients experience episodes of erythema, skin flushing, telangiectasia, and papulopastular cutaneous eruptions. Inflammation of the eyes and eyelids also develop and leads to the formation of chalazion. $^{25,26}$

\section{Exposure to air pollutants}

Chalazion may develop after exposure to air pollutants. Foreign substances can occlude the Meibomian gland duct leading to obstruction of the sebum and the development of chalazion. This can affect all individuals at any age, of either sexes, and irrespective of their race. It is specifically common when poor hygiene and lack of regular cleansing of the eyes exist. ${ }^{1}$

\section{Poor eyelid hygiene}

Poor hygiene of the eyelids was reported to be a significant risk factor for chalazion formation. ${ }^{13}$ Regular cleansing of the eyelids seems to be essential for removing any foreign bodies, debris, or cosmetic agents occluding Meibomian glands ducts. This may also explain the high prevalence of chalazion among females using eye cosmetics. ${ }^{27}$

\section{Chronic blepharitis}

Blepharitis is another established predisposing factor for chalazion. ${ }^{13}$ Blepharitis is associated with inflammation of the Meibomian glands and their ducts leading to occlusion and sequestration of sebaceous secretions. The risk for chalazion formation with blepharitis increases with poor eyelid hygiene, seborrheic dermatitis, and hyperimmunoglobulin E states. 


\section{Seborrheic dermatitis}

Seborrheic dermatitis is a generalized cutaneous chronic inflammatory disease affecting various areas of the body. Areas with sebaceous glands are particularly affected. Meibomian glands of the eyelids, being secretory sebaceous glands are often affected participating in seborrheic blepharitis and chalazion formation. ${ }^{28}$

\section{Hyperlipidemia}

Hyperlipidemia is associated with increased secretion of the sebum from the meibomian glands. ${ }^{13}$ The viscous sebum occludes the meibomian gland ducts leading to sequestration of the secretion and cystic formation. Hyperlipidemia also increases the risk for blepharitis and subsequently chalazion. ${ }^{14}$

\section{Eyelid trauma}

Eyelid trauma causes a disruption to the structure of the meibomian gland ducts. Blunt injury to the tarsal plate leads to occlusion of the ductal lumen, sebum sequestration, and subsequently chalazion formation. Similarly, sharp eyelid trauma can lead to direct injury to the ducts and malfunction of the ductal release of the produced sebum in the meibomian glands. ${ }^{14,27}$

\section{Other predisposing factors}

Immunodeficiency states and hyperimmunoglobulin $\mathrm{E}$ syndrome (Job syndrome) were reported to be associated with chalazion. ${ }^{1,8}$ Less well-established predisposing factors for Chalazia include exposure to ultraviolet rays, use of eyelid cosmetics, dry eye, trachoma, and stress. However, the mechanisms of these factors are still unknown.

\section{CONCLUSION}

Chalazion is one of the most common cystic lesions affecting the eyelids. Although many cases are tiny or small in size, some are large enough to cause significant pressure on eye globe ad visual obscuration. The exact incidence of chalazion is variable among the studies and it differs depending on certain predisposing factors. The incidence ranges from 0.2 to $0.7 \%$ with females being slightly more affected. The main predisposing factors to chalazion formation include hormonal changes during puberty and pregnancy, viral and bacterial infection, rosacea, exposure to air pollutants, poor eyelid hygiene, chronic blepharitis, seborrheic dermatitis, eyelid trauma, immune deficiency states, eyelid trauma, hyperlipidemia, tuberculosis, and leishmaniasis.

Funding: No funding sources Conflict of interest: None declared Ethical approval: Not required

\section{REFERENCES}

1. Arbabi EM, Kelly RJ, Carrim ZI. Chalazion. Praxis (Bern 1994). 2011;100(8):479-80.

2. Unal M. Chalazion treatment. Orbit. 2008;27(6):397-8.

3. Driver PJ, Lemp MA. Meibomian gland dysfunction. Surv Ophthalmol. 1996;40(5):343-67.

4. Donaldson MJ, Gole GA. Amblyopia due to Inflamed Chalazion in a 13-Month Old Infant. Clin Experimental Ophthalmol. 2005;33:332-3.

5. Khurana AK, Ahluwalia BK, Rajan C. Chalazion therapy. Acta Ophthalmol. 2009;66(3):352-4.

6. Devlioti D, Tsintarakis T, Milioti G, Seitz B, Kasmann-Kellner B. Unusual course of a chalazion. Ophthalmologe. 2015;112(3):269-71.

7. Chang M, Park J, Kyung SE. Extratarsal presentation of chalazion. Int Ophthalmol. 2017;37(6):1365-7.

8. Al-Faky YH. Epidemiology of benign eyelid lesions in patients presenting to a teaching hospital. Saudi J Ophthalmol. 2012;26(2):211-6.

9. Kersten RC, Ewing-Chow D, Kulwin DR, Gallon M. Accuracy of Clinical Diagnosis of Cutaneous Eyelid Lesions. Ophthalmology. 1997;104(3):47984.

10. Hsu HC, Lin HF. Eyelid tumors in children: A clinicopathologic study of a 10-year review in Southern Taiwan. Ophthalmologica. 2004;218(4):274-7.

11. Ni Z. Histopathological classification of 3,510 cases with eyelid tumor. Zhonghua Yan $\mathrm{Ke} \mathrm{Za} \mathrm{Zhi.}$ 1996;32(6):435-7.

12. Mindy Ann Smith AEMJ. Hordeolum and Chalazion. In: The Color Atlas of Family Medicine. Second. McGraw-Hill; 2013.

13. Kumar J, Pathak AK, Verma A, Dwivedi S. Study of Incidence And Risk Factors of Chalazion in Bundelkhand Region. IOSR J Dent Med Sci. 2017;16(5):5-8.

14. Otulana T, Bodunde O, Ajibode H. Chalazion, a Benign Eyelid Tumour - The Sagamu Experience. Niger J Ophthalmol. 2009;16(2).

15. Mansour AM, Chan CC, Crawford MA, et al. Virusinduced chalazion. Eye. 2006;20(2):242-6.

16. Mustafa TA, Oriafage IH. Three methods of treatment of chalazia in children. Saudi Med J. 2001;22(11):968-72.

17. D'hermies F, Fayet B, Meyer A. Chalazion Mimicking an Eyelid Tumor. Journal francais d'ophtalmologie. 2004;27:202-5.

18. Mueller JB, McStay CM. Ocular Infection and Inflammation. Emerg Med Clin North Am. 2008;26(1):57-72.

19. Aoki M, Kawana S. Bilateral chalazia of the lower eyelids associated with pulmonary tuberculosis [8]. Acta Derm Venereol. 2002;82(5):386-7.

20. Mohammadpour I, Motazedian MH, Handjani F, Hatam GR. Cutaneous leishmaniasis of the eyelids: A case series with molecular identification and 
literature review. Korean J Parasitol. 2016;54(6):787-92.

21. Mittal R, Tripathy D, Sharma S, Balne PK. Tuberculosis of eyelid presenting as a chalazion. Ophthalmology. 2013;120(5).

22. Lai JJ, Chang P, Lai KP, Chen L, Chang C. The role of androgen and androgen receptor in skin-related disorders. Arch Dermatol Res. 2012;304(7):499510.

23. Imperato-Mc Ginley J, Gautier T, Cai LQ, Yee B, Epstein J, Pochi P. The androgen control of sebum production. Studies of subjects with dihydrotestosterone deficiency and complete androgen insensitivity. J Clin Endocrinol Metab. 1993;76(2):524-8.

24. DeStafeno JJ, Kodsi SR, Primack JD. Recurrent Staphylococcus aureus chalazia in hyperimmuno- globulinemia e (Job's) syndrome. Am J Ophthalmol. 2004;138(6):1057-8.

25. Chosidow O, Cribier B. Epidemiology of rosacea: Updated data. In: Annales de Dermatologie et de Venereologie. 2011: 138.

26. van Zuuren EJ. Rosacea. N Engl J Med. 2017;377(18):1754-64.

27. Sharma S. Ophthaproblem. Chalazion. Can Fam physician M. 1998;44.

28. Eltis M. Seborrheic blepharitis. Clin Refract Optom. 2012;23(3):74-7.

Cite this article as: Alsammahi A, Aljohani Z, Jaad N, Daia OA, Aldayhum M, Almutairi M, et al. Incidence and predisposing factors of chalazion. Int $\mathbf{J}$ Community Med Public Health 2018;5:4979-82. 